RUNNING HEAD: Tiny Dancers



To appear in Developmental Psychology

(C) 2022, American Psychological Association. This paper is not the copy of record and may not exactly replicate the final, authoritative version of the article. Please do not copy or cite without authors' permission. The final article will be available, upon publication, via its DOI: 10.1037/dev0001363

\section{Tiny dancers: Effects of musical familiarity and tempo on children's free dancing} Haley E. Kragness, Farhat Ullah, Emma Chan, Rachel Moses, Laura K. Cirelli University of Toronto Scarborough, Department of Psychology, Toronto, ON, Canada

Acknowledgements: Our thanks to Lucy Anderson, Joey Ball, Ester Chow, Matthew Eitel, Lanyi Lin, Marisa Oliveros and Mikayla Samuel for their involvement in participant recruitment, data collection, and video annotation.

Author Note: Video data collected from participants are available to authorized researchers via Databrary (volume 1141; Kragness et al., 2020). Data and analysis codes are available by emailing the corresponding author. This study's design and analysis were not pre-registered. 


\section{Abstract}

2 Around the world, musical engagement frequently involves movement. Most adults easily clap

3 or sway to a wide range of tempos, even without formal musical training. The link between

4 movement and music emerges early -young infants move more rhythmically to music than

5 speech, but do not reliably align their movements to the beat. Laboratory work encouraging

6 specific motor patterns (e.g., drumming, tapping) demonstrates that toddlers and young

7 children's movements are affected by music in a rudimentary way, such that they move faster to

8 faster rhythms (tempo flexibility). In the present study, we developed and implemented a novel

9 home recording method to investigate how musical familiarity and tempo affect children's

10 naturalistic free-dance movements. Caregivers made home recordings of their children's

11 responses to an experimenter-created playlist $(N=83$, age range $=1.25$ to 3.91 years, $M$ age $=$

122.39 years, $S D=0.74$ years; 41 girls, 42 boys; $75 \%$ of household incomes $>\$ 90000 \mathrm{CAD}$ ).

13 Children listened to 1-min excerpts of their favorite music and unfamiliar, genre-matched music,

14 each played at 90, 120, and 150 bpm (pitch constant; order randomized). Children moved faster

15 to faster music and demonstrated tempo flexibility for both favorite and unfamiliar music.

16 Favorite music encouraged more smiling across tempo conditions than unfamiliar music, as well

17 as more dancing in the slowest tempo condition. Results demonstrate that young children's self-

18 selected movements are affected by musical tempo and familiarity. We also demonstrate the

19 usefulness of a naturalistic home recording method for assessing early auditory-motor

20 integration.

21 Keywords: music, rhythm, movement, dance, development, children 

9 Trehub et al., 2015). 2021).

\section{Introduction}

Movement is a widespread feature of musical engagement. This can be seen when we inadvertently tap along to a song on the radio, rock an infant while gently singing a lullaby, or perform choreographed dance routines. Neural and behavioral evidence underscore the involvement of motor processes in rhythm perception, even in the absence of overt movement (Cannon \& Patel, 2021; de Kock et al., 2021; Grahn \& Brett, 2007; Gordon et al., 2018; Manning \& Schutz, 2013; Morillon \& Baillet, 2017). Indeed, movement is considered an inseparable component of musical engagement in many cultures (Savage et al., 2015; Bohlman, 2002;

Most adults easily move in synchrony with a musical beat (the underlying isochronous pulse in a piece of music). Though beat synchronization often feels effortless, it requires a complex interplay between auditory and motor systems (Patel \& Iverson, 2014). Accordingly, rhythm perception and musical synchronization abilities develop slowly and may not reach adult levels until late childhood or adolescence (McAuley et al., 2006; Nave-Blodgett et al., 2020). However, the link between movement and music is observable in early infancy (Philips-Silver \& Trainor, 2005; Zentner \& Eerola, 2010). Some precocious infants display the urge to move to music just a few months after birth (Fujii et al., 2014), and most North American caregivers report that their infants move in response to music by 8 months of age (Kim \& Schachner,

The ability to move faster to faster music and slower to slower music, tempo flexibility, is thought to be an important prerequisite for auditory-motor synchronization (e.g., Zentner \& Eerola, 2010). In the lab, children exhibit tempo flexibility well before they are able to synchronize reliably to music. In one early laboratory study, 5- to 24-month-olds produced faster movements to faster music and rhythms (Zentner \& Eerola, 2010). More recently, Rocha and Mareschal (2017) provided 10- and 18-month-old infants with handheld sleigh bells while they listened to a series of lively pop music (e.g., Jennifer Lopez' Let's Get Loud). Neither age group 
1 showed strong evidence of synchronization, but the older infants displayed more tempo

2 flexibility than the younger infants. Yu and Myowa (2021) asked 18-, 30-, and 42-month-olds to

3 tap on a set of toy drums to Twinkle, Twinkle, Little Star. Children in all three age groups tapped

4 faster when the music was faster, although the youngest children hit the drum less frequently,

5 showed greater variability, and were further from the target tempo in the slow condition,

6 especially compared to the older children. Other laboratory research has investigated children's

7 ability to tap their finger on a touch-screen device or to strike a drum using a drumstick along to

8 isochronous drumbeats without musical accompaniment. While 2.5-year-old children show

9 some tempo flexibility, especially when synchronization is modelled by a social partner or

10 reinforced by interesting visual displays, in general, substantial improvements are observed

11 between 2.5 and 4 years (Provasi \& Bobin-Bègue, 2003; Kirschner \& Tomasello, 2009).

12 Familiarity is potentially an important factor in early musical movements. In laboratory

13 studies, infants preferentially listen to and are soothed by familiar over unfamiliar songs (Cirelli

14 \& Trehub, 2020; Kragness, Johnson, \& Cirelli, 2022). Previous studies of musical beat

15 synchronization have largely used familiar music (but see Rocha \& Mareschal, 2017), assuming

16 that a boost in task enjoyment would facilitate optimal performance. Results from a recent case

17 study comparing one child's responses to familiar and unfamiliar music stand in contrast to that

18 view. Researchers implemented a home-based method for exploring early musical movements

19 with 19-month-old "V" (Cirelli \& Trehub, 2019). V was recorded at home while she listened to 1-

20 min clips of two familiar pieces of music and two unfamiliar pieces of music over several weeks.

21 Each piece of music was presented at its original tempo (close to 120 beats per minute, bpm)

22 and at a faster (140 bpm) and slower (100 bpm) tempo without modulating pitch. $V$ danced

23 more to familiar music and smiled more frequently when she danced. Notably, $V$ showed better

24 tempo matching when listening to unfamiliar compared to familiar music. Familiar music,

25 regardless of tempo, elicited fast dancing. 
Much of what is known about early musical movements stems from laboratory research

2 investigating specific target movements, like clapping, tapping, or drumming. Henry and

3 colleagues (2021) recently argued that a comprehensive investigation of synchronization

4 abilities across the animal kingdom must consider the body parts and motivational goals that

5 apply to each species, which can be achieved by combining traditional lab work with

6 observational field studies. A similar approach will be crucial for understanding age-related

7 differences in movement to music. To our knowledge, only one previous study has used a free

8 dancing methodology to examine children's auditory-motor integration (Eerola et al., 2006).

9 Researchers used motion capture to measure the properties of 2- to 4-year-old children's free

10 dancing in a lab. Though children's movements were at times periodic, their movements were

11 unaffected by manipulations of the musical tempo. Moreover, many of the children (18 of the 46

12 tested) refused to dance at all. It is likely that children would be more motivated to engage in

13 musical behaviors in familiar settings, such as their own home. Indeed, North American

14 preschool children often spontaneously integrate musical movement in their play at home (Cirelli

15 et al., 2020). Home-based assessments of singing proficiency and pitch range in toddlers and

16 preschoolers reveal that laboratory measures may underestimate early musical abilities

17 (Gudmundsdottir \& Trehub, 2018). Thanks to technological advancements, home-based

18 methods are gaining popularity in developmental research, providing windows into a variety of

19 behaviors such as play (Herzberg et al., 2021), language acquisition (Bergelson et al., 2018),

20 and visual attention (Slone et al., 2018).

21 The purpose of the present study was to examine the effects of tempo and musical

22 familiarity on 1- to 4-year-old children's free dancing using a novel home-recording method. By

23 asking children to dance freely at home, we hoped to provide a context conducive to children's

24 comfort and motivation. Caregivers were provided with a custom playlist including 1-min

25 excerpts of their child's favorite music and genre-matched unfamiliar music at three tempos (90,

26 120, and $150 \mathrm{bpm})$. Caregivers played the playlist for their child at home while recording videos 
1 (see Supplemental Material for video examples). Videos were annotated to identify when

2 children danced, movement tempos, and when they smiled. We predicted that (1) children

3 would free dance faster to faster music, and (2) tempo flexibility would increase with age. We

4 additionally explored whether musical familiarity modulated children's movement rate and tempo

5 matching. If the rate of children's dancing was more congruent with the metrical hierarchy for

6 favorite music, this would suggest that motivational features and/or familiarity with musical

7 features might scaffold tempo matching. In contrast, excited responses to the favorite music

8 could inhibit children's tempo flexibility by encouraging universally fast movements, as observed

9 in the case study results with toddler V (Cirelli \& Trehub, 2018). Finally, we predicted that

10 children would dance and smile more to favorite than unfamiliar music.

\section{Methods}

\section{Participants}

Caregivers $(n=210)$ first participated in the Favorite Music Survey. Families with

15 children 1.25 to 3.9 years old were recruited via social media, recruitment websites (Children

16 Helping Science), and the UTSC iKids Centre family database. After filling out an online

17 questionnaire, families were invited to participate in the Dance Phase portion of the study.

18 Videos were received from 92 participants. This final $N$ was determined by the number of

19 children that could be recruited for this study in one year, and efforts were made to recruit

20 evenly across the age range. One participants' video was lost due to technical difficulties, and

21 three additional participants' data were omitted due to experimenter error in creating their

22 stimuli. Two participants' caregivers indicated that this was the second recording of the dance

23 session, thus familiarizing their children with the "unfamiliar" tunes, and trials in which caregivers

24 danced in frame with their child were also omitted (8.2\% of trials, three additional participants).

25 After these exclusions, a total of 83 participants remained (age range $=1.25$ to 3.91 years, $M$

26 age $=2.39$ years, $S D=0.74$ years, 41 girls, 42 boys; 81 children from Canada, 1 from the USA, 
11 from the UK). Household income varied, and most families were economically wealthier than

2 the average family in Toronto, Canada (45\% reporting $>\$ 120,000 \mathrm{CAD} /$ year, $40 \%$ reporting

3 between $\$ 60,000$ and $\$ 120,000 /$ year, $13 \%$ reporting $<\$ 60,000 /$ year, 2 families did not

4 respond). As a token of appreciation, families received a Junior Scientist certificate for each

5 completed phase and were entered into a gift certificate draw (1 in 20 chance at a card valued

6 at $\$ 30 \mathrm{CAD}$ ). All procedures were approved by the University of Toronto Research Ethics Board

7 (Protocol 36642, Adult, children and infant music perception and engagement).

8

$9 \quad$ Stimuli 21 that song.

In the initial survey, caregivers were asked to indicate up to five of their child's favorite pieces of music, including the title and artist (if applicable), and a rating of how often their child hears the music on a scale of 1-rarely to 5-multiple times each day (four caregivers provided music, but did not provide frequency ratings on their surveys). From this list, an experimenter selected one piece of music to designate as the "favorite" in this study, using their judgment to select music that was both frequently heard and easily sourced for the experiment. For most of the children (74 of the 79), the selected "favorite" was the music the child heard most frequently, according to caregiver report. A specific favorite version (recording by a specific artist) could be identified for 70 of the 83 participants. The remaining participants' favorite music were wellknown children's songs with no specified artist (e.g., "Wheels on the Bus", "Old McDonald had a Farm"). For these 13 participants, the experimenter used a recorded child-directed version of

The "unfamiliar" music was a pre-selected genre-matched recorded piece of music (see Supplemental Material). A research assistant used their best judgment to determine the tempo of the original music by tapping along to the music using an online bpm tool (www.beatsperminuteonline.com). The average tempo of favorite music was $115 \mathrm{bpm}$ (SD = 25 bpm). Excerpts from both the "favorite" and "unfamiliar" music were edited in Audacity software 
1 v2.3.0 (Audacity Team, 2018) to manipulate tempo while maintaining the pitch. The music was

2 edited to three different tempos $(90,120$, and $150 \mathrm{bpm})$. Unless the music had an original

3 tempo of either 90,120 , or $150 \mathrm{bpm}$, all three versions were modified from the original. The

4 music was then cropped to excerpts one minute of length starting either when lyrics began or,

5 for music without lyrics, at the beginning of the recording. This was done to ensure recognition

6 of the music with lyrics. Finally, the 1-min excerpts were compiled into a playlist with 5 s of

7 silence between each of the 6 excerpts, with a brief fade-in and fade-out for each excerpt.

8 Playlists were pseudo-randomly ordered such that the favorite and unfamiliar music alternated,

9 but tempo order was randomized. These custom playlists were uploaded to YouTube.com and 10 shared with the caregiver.

Procedure

If caregivers indicated interest in the Dance Phase at the end of the Favorite Song

14 Survey, the experimenter sent clips of the music to caregivers via email to listen to (without their 15 child present) to confirm that the child knew the "favorite" music and that they did not know the 16 genre-matched "unfamiliar" music. The experimenter then sent the custom playlist to the family.

17 Caregivers were given the option to record video on their own $(N=70)$, or to schedule an 18 appointment with an experimenter through video call so that video could be recorded via screen 19 capture $(N=13)$. All caregivers were asked to prepare an open, safe area for their child to 20 move around in before beginning the experiment, and to avoid dancing themselves or 21 influencing their child's behavior, especially while the music was playing. General words of 22 encouragement were permitted (e.g., "Great job!").

23 After the Dance Phase, caregivers completed a demographics questionnaire, which

24 included questions about children's history participating in dance/musical movement classes.

25 Caregivers were also asked to complete the Music@Home Preschool questionnaire (Politimou 26 et al., 2018), which captures information about children's home musical environment including 
1 parental beliefs about the importance of music in early childhood, children's engagement with

2 music, parental initiation of musical behavior, and the breadth of musical exposure children

3 experience at home. Neither experience with dance classes or scores on the Music@Home (or

4 corresponding subscales) significantly correlated with any dependent measure (see

5 Supplemental Materials).

6 Behavioral Annotation. A research assistant divided the video recording into six 1-min

7 excerpts corresponding to times when the music was being presented to the participants and

8 uploaded these trial-level videos to Databrary (volume 1141, Kragness et al., 2020). Trained

9 annotators downloaded the videos from Databrary and annotated behaviors using ELAN

10 Linguistic Annotator (https://tla.mpi.nl/tools/tla-tools/elan, Lausberg and Sloetjes, 2009).

11 Videos were played back silently to ensure that annotators were naïve to condition. For

12 each video, annotators first notated (1) bouts of dancing. Dance bouts were defined as windows

13 of time in which the child was subjectively judged to be moving because of the music, and not

14 for another obvious reason. Within dance bouts, they next identified (2) rhythmic bursts, or

15 bursts of movements that had an identifiable tempo (e.g., the same movement was repeated

16 two or more times). Within these bursts, annotators indicated (3) the onset of each movement

17 belonging to the same burst, defined as the most discrete component of the rhythmic movement

18 (e.g., if a child clapped three times, the moments when their hands connected each time were

19 identified; if they alternated between stepping on their left foot and then their right, the moments

20 when their left foot connected with the ground were identified). For expressions of joy,

21 annotators indicated windows of time when (1) the child's facial expressions were visible and (2)

22 expressions of positive affect were evident (i.e., smiling, laughing). Two annotators identified

23 facial visibility and positive affect, and two completed movement annotation. Interrater

24 agreement for 18 videos (all six videos from three children, pseudo-randomly selected such that

25 all three contained all behaviors of interest) was high for duration of face visibility (Pearson's $r$ 
$1=.98)$, duration of expressions of joy $(r=.87)$, duration of dancing $(r=.98)$, and average inter-

2 onset interval between repeated movements in a trial $(r=.92)$.

3 Secondary video coding was done with audio on. Research assistants indicated (1) how

4 long children were completely out of the video frame, and (2) how often caregivers specifically

5 asked children to dance. Results from a mixed effects model did not indicate any evidence that

6 caregivers' requests to dance differed across tempo or familiarity conditions ( $p$ 's > .330), so

7 trials containing such requests were retained in the full analysis. Independent coding of $16 \%$ of

8 the trials yielded high interrater reliability for the 3 measures (time off frame, $r=0.94$; caregiver

9 verbal encouragement, $92.8 \%$ trial-level agreement; caregiver dance requests, $88.5 \%$ trial-level 10 agreement).

11 Analyses. Linear mixed effects models (LMEM; glmmTMB package, Brooks et al.,

12 2017) in R (version 3.6.3, R Core Team, 2020) were used to evaluate the effects of familiarity

13 and tempo on children's behavior. The dependent measures were (1) median movement tempo

14 (calculated from inter-movement intervals in rhythmic burst coding), (2) temporal

15 correspondence (calculated from inter-movement intervals and described in more detail below),

16 (3) proportion of time dancing (sum of dance bout times/time in frame), and (4) proportion of

17 time smiling (total time smiling/time their face was visible). We contrast-coded the familiarity

18 factor (favorite $=1$, unfamiliar $=-1$ ). Tempo was treated as an ordinal variable, and we tested

19 linear effects. For each model, we first tested whether including any interactions

20 (age familiarity ${ }^{\star}$ tempo, age ${ }^{\star}$ familiarity, age*tempo, or familiarity ${ }^{\star}$ tempo) significantly improved

21 model fit, and removed it from the model when it did not (see Supplemental Material for model

22 specifications and output). Random intercepts were included in the models for participant

23 identity to account for repeated measures, but random slopes were not included due to failure to

24 converge and/or failure to improve model fit. Finally, age was included as a continuous

25 predictor. For proportion data (temporal correspondence, proportion of time dancing,

26 expressions of joy) we used a beta distribution for the outcome variables, and included zero- 
1 inflation when it significantly improved the model. For non-proportion data (median movement

2 tempo), we used a Gaussian distribution for the outcome variable.

Transparency and openness. Video data are available to authorized researchers via

4 Databrary (volume 1141, Kragness et al., 2020). Sample size determination, all exclusions and

5 all manipulations are reported above. Data and analysis codes are available via the Open

6 Science Framework (Kragness, Ullah, Chan, Moses, \& Cirelli, 2022; https://osf.io/q8zjy/). Data

7 were analyzed using R (version 3.6.3, R Core Team, 2020). This study's design and analysis

8 were not pre-registered.

\section{Results}

Out of 83 children, 10 never danced ( $12 \%)$, and $64 \%$ of trials overall contained dancing. When they danced, they often did so for a significant portion of the 1-min excerpt $(M=$ $43 \%, S D=30 \%)$.

\section{Tempo Flexibility}

Inter-movement-intervals were calculated as the time between maximal extensions belonging to the same rhythmic burst (in other words, between-burst inter-movement-intervals were omitted) and converted to a rate (beats per minute).

To obtain a movement tempo measurement for each trial, we used the median movement tempo. Identical statistical results were observed if we used the mean tempo instead of the median tempo. Children for whom no rhythmic bursts were identified were omitted from this analysis, leaving 73 children. Children demonstrated tempo flexibility: median movement tempo increased linearly with musical excerpt tempo, $B=13.308, S E=3.776, z=3.525, p<$ .001 . Older children danced at a slower tempo than younger children, $B=-13.665, S E=6.157$, $Z=-2.219, p=.026$, and children danced faster in the favorite vs. unfamiliar condition, $B=$ 4.495, $S E=2.200, z=2.044, p=.041$. On average, children's median dance tempo was 104 bpm in the context of favorite music, compared to $97 \mathrm{bpm}$ in the context of unfamiliar music (Figure 1; see Supplemental Table 1). 
To further explore whether age related to tempo flexibility, median movement bpm

2 difference scores were calculated for the fastest (150 bpm) compared to the slowest (90 bpm)

3 trials. We predicted that older children would have a larger difference score in median

4 movement bpm across these two tempo conditions than younger children. Children with

5 rhythmic bursts for at least one of the fastest and slowest excerpts were included in the

6 analysis, leaving 53 children. This analysis did not reveal a significant association between age

$7 \quad$ and tempo flexibility, $r(51)=.051, p=.717$.

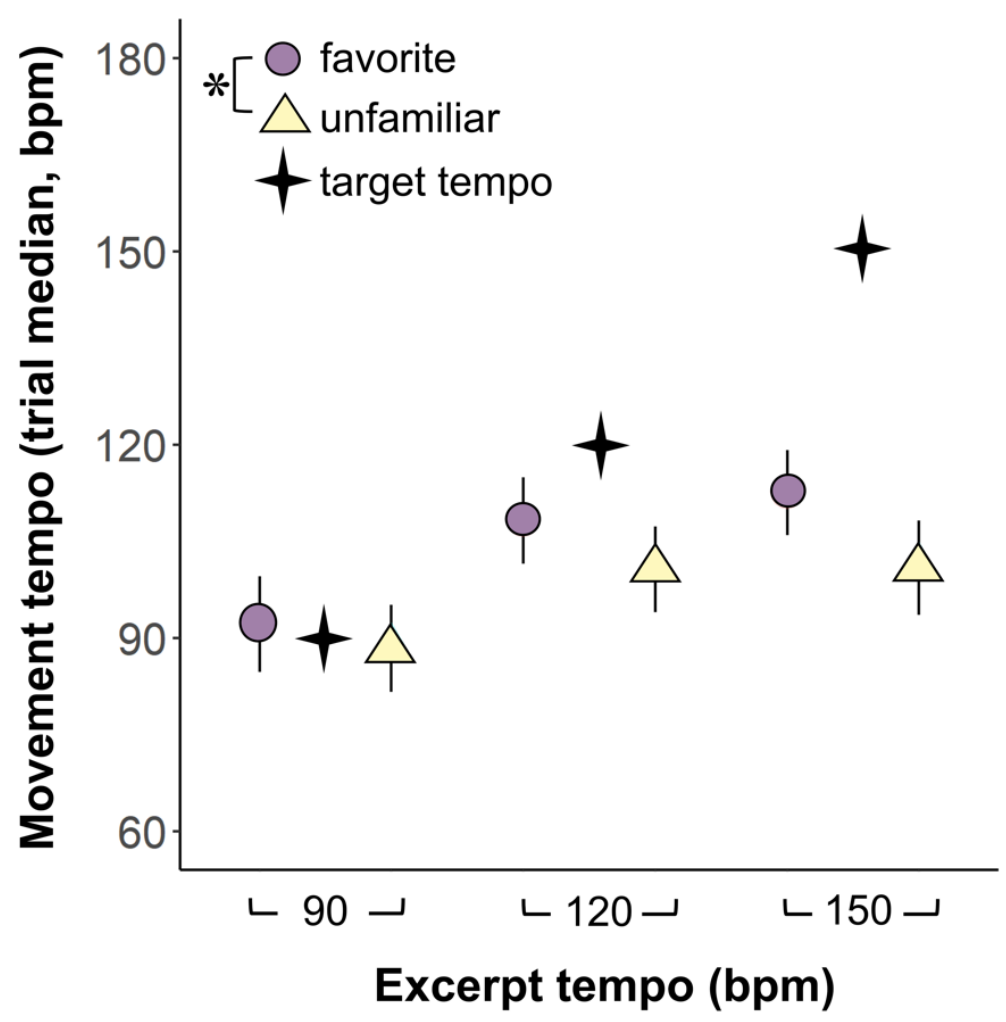

8

9 Figure 1. Depicts the average movement tempo of children's rhythmic movements (each trial's 10 median bpm). Error bars are 95\% within-subject confidence intervals. Black stars signify the 11 excerpt tempo.

12

\section{Temporal Correspondence}


The previous analysis demonstrated that on average, children's movements were faster

2 when the music was faster. Next, we assessed whether children's movements were closer to

3 the excerpt tempos at different levels of tempo and familiarity. To do this, we used a measure

4 we call "temporal correspondence", which the reflects the proportion of a children's movements

5 that are at a rate near any of the metrical levels of the musical excerpts. To assess whether

6 children were more successful at matching the temporal structure for favorite compared to

7 unfamiliar music, we used inter-movement-intervals to calculate a temporal correspondence

8 measure per trial. This measure represents the percentage of rhythmic movements that

9 corresponded to any level of the musical meter for each excerpt (Kragness, Anderson, Chow,

10 Schmuckler, \& Cirelli, 2022). For each tempo condition, all inter-movement-intervals falling

11 within $a+/-10 \%$ window around the beat rate (i.e., target tempo), half beat rate, or double beat

12 rate were considered to be temporally correspondent. On average, $28 \%$ of children's

13 movements fell within these windows $(S D=15 \%)$. To receive a temporal correspondence

14 score, children needed to produce a minimum of 10 rhythmic movements total in that trial (not

15 necessarily consecutive). This left 57 children in the analysis. We found no effect of age,

16 musical familiarity, or tempo on temporal correspondence scores (all p's > .372; see Figure 2

17 and Supplemental Table 2).

While our planned hypotheses considered linear effects of tempo, visualizing temporal

20 reflective of the data, especially for the favorite music condition. In an exploratory analysis, we

21 tested a model including a tempo (quadratic) by familiarity interaction, which reached

22 significance, $B=0.244, S E=0.101, z=2.408, p=.016$ (note, however, that a direct

23 comparison of the models with and without an interaction term only provided tentative support

24 for including the interaction, $p=.051$ ). To break down this interaction, we explored the quadratic

25 effect of tempo for each level of musical familiarity separately. There was no effect of tempo for

26 unfamiliar music $(p=.634)$. In the favorite music condition, there was a significant quadratic 
1 effect of tempo such that children had the lowest temporal correspondence scores for the 120

2 bpm condition, $B=0.449, S E=0.135, z=3.326, p<.001$ (see Supplemental Table 3). Given

3 the exploratory nature of this analysis, we caution against over-interpretation.

$4 \quad$ For the favorite music, we next asked whether their temporal correspondence scores

5 were affected by how close the tempo of the music was, compared to the original tempo of the

6 music. Unfamiliar excerpts were omitted for this analysis, given that children should not have

7 any particular expectations for the tempo of music they have never heard. Common songs that

8 did not have a definitive recorded version associated with them were likewise omitted. Temporal

9 correspondence was numerically higher for songs further from the original tempo, but this

10 association did not reach significance, $B=0.008, S E=0.004, z=1.840, p=.066$. 


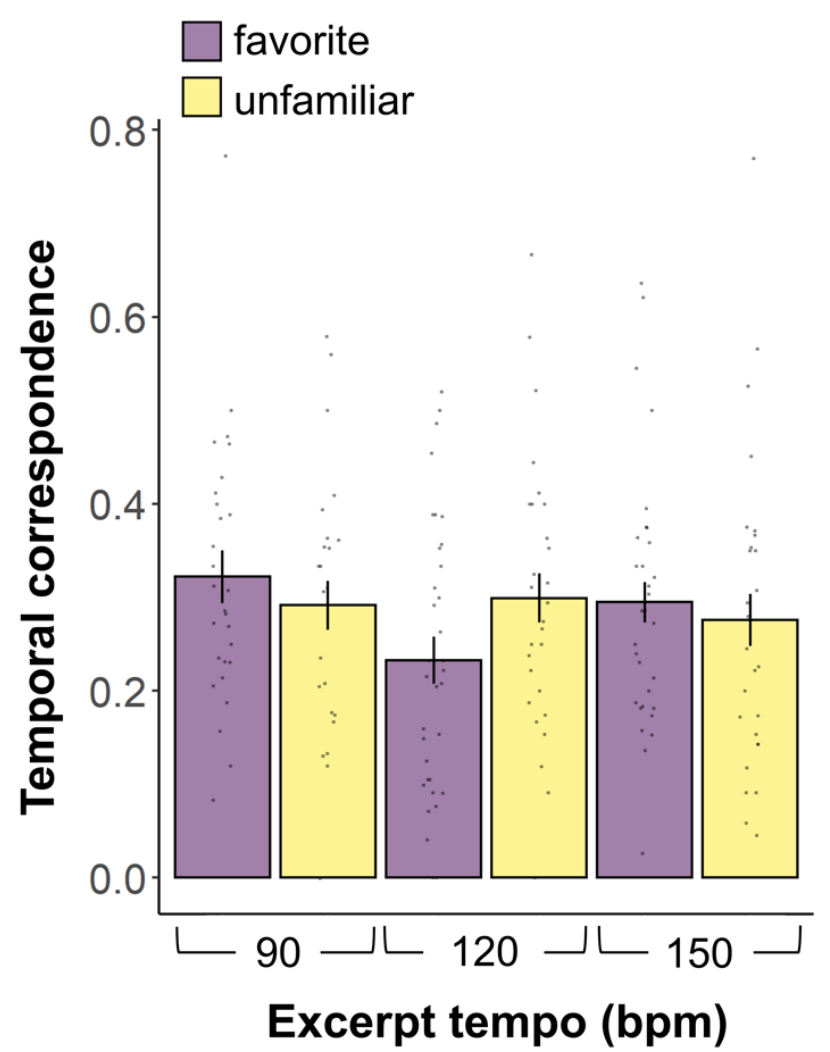

2 Figure 2. Depicts the proportion of rhythmic movements that corresponded to one of three 3 metrical levels of the musical excerpt in that trial (beat rate, half beat rate, or double beat rate). 4 Movements were considered temporally correspondent if they fell within a $+/-10 \%$ window of 5 those rates. Individual points represent individual participants, bars represent group averages, 6 and error bars represent 95\% within-subject confidence intervals.

\section{Proportion of Time Dancing}

There was a significant effect of age on the proportion of time children danced, such that

12 older children danced more, $B=0.883, S E=0.132, z=6.676, p<.001$. There was also a

13 significant interaction between tempo and familiarity, $B=-0.197, S E=0.095, z=-2.088, p=$

14.037 (see Figure 3 and Supplemental Table 4). To break down the interaction, we investigated 
1 the effect of familiarity separately in each tempo condition. In the $90 \mathrm{bpm}$ condition, children

2 spent significantly more time dancing to the favorite music $(\sim 30 \%)$ than the unfamiliar music

$3(\sim 22 \%), B=0.245, S E=0.096, z=2.548, p=.011$. There was no significant difference in the

4 proportion of time children spent dancing to favorite vs. unfamiliar music in the $120 \mathrm{bpm}$ or 150

5 bpm conditions, $p$ 's $>.426$.

6 As with temporal correspondence, we asked whether dancing was affected by how close

7 the tempo of the music was, compared to the original tempo. Unfamiliar excerpts were omitted

8 for this analysis, given that children should not have any particular expectations for the tempo of

9 music they have never heard. Common songs that did not have a definitive recorded version

10 associated with them were likewise omitted. Consistent with the lack of effect in tempo above,

11 there was no significant effect on the proportion of time dancing based on whether the music

12 was closer to the "correct" tempo $(p=.793)$. 


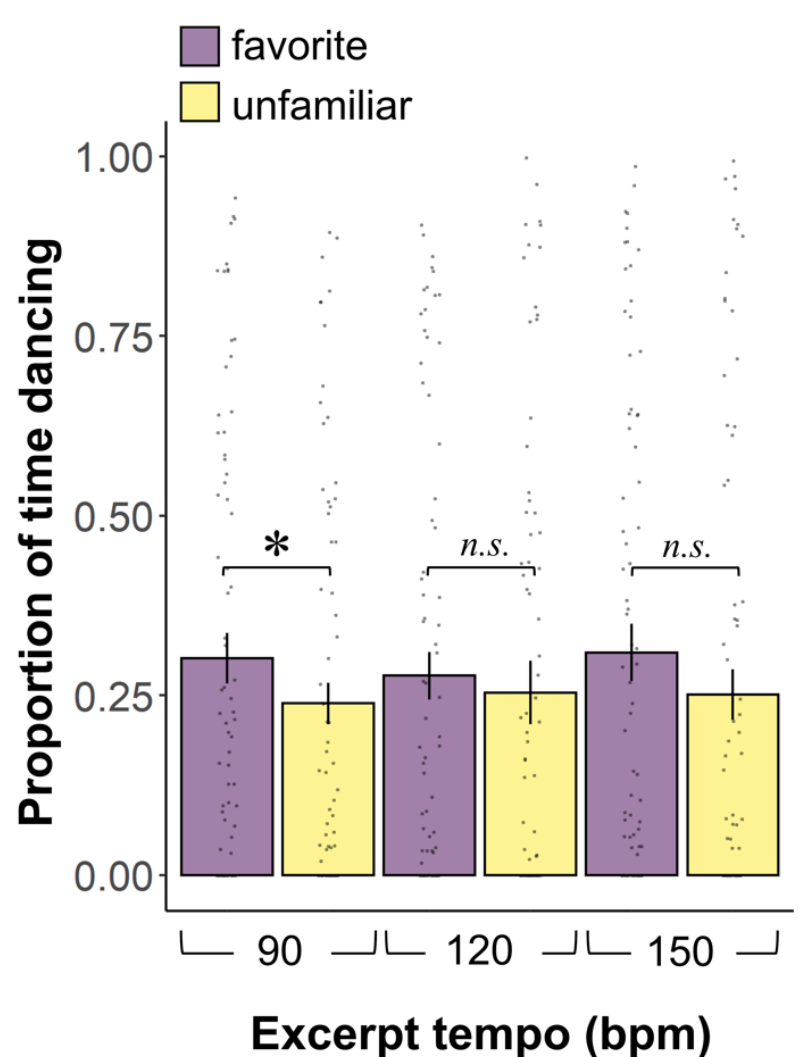

2 Figure 3. Depicts the proportion of time participants spent dancing. Individual points represent 3 individual participants, bars represent group averages, and error bars represent 95\% within4 subject confidence intervals. ${ }^{*} p<.05$

\section{Expressions of Joy}

Expressions of joy (smiles or laughter) were identified in $55 \%$ of trials. For proportion of

8 time spent smiling, we found no significant linear effect of tempo $(p=.221)$ nor effect of age $(p=$

9 .389), but there was a significant effect of familiarity, $B=0.094, S E=0.048, z=1.977, p=.048$

10 (Figure 4; see Supplemental Table 5). In aggregate, children smiled about $10 \%$ of the time

11 during favorite music, and about $8 \%$ of the time during unfamiliar music.

For the favorite music, we next asked whether children's expressions of joy were

13 affected by how close the tempo of the music was, compared to the original tempo of the music. 
1 Unfamiliar excerpts were omitted for this analysis, given that children should not have any

2 particular expectations for the tempo of music they have never heard. Common songs that did

3 not have a definitive recorded version associated with them were likewise omitted. Similarity to

4 the original tempo was not a significant predictor of expressions of joy, $B=0.004, S E=0.004, z$

$5=1.203, p=.229$.

6 Several previous studies of musical movement observed associations between dancing

7 and smiling (see Introduction). Here, too, trials with higher proportions of time spent dancing

8 also had higher proportions of time expressing joy, $B=1.155, S E=0.205, z=5.637, p<.001$.

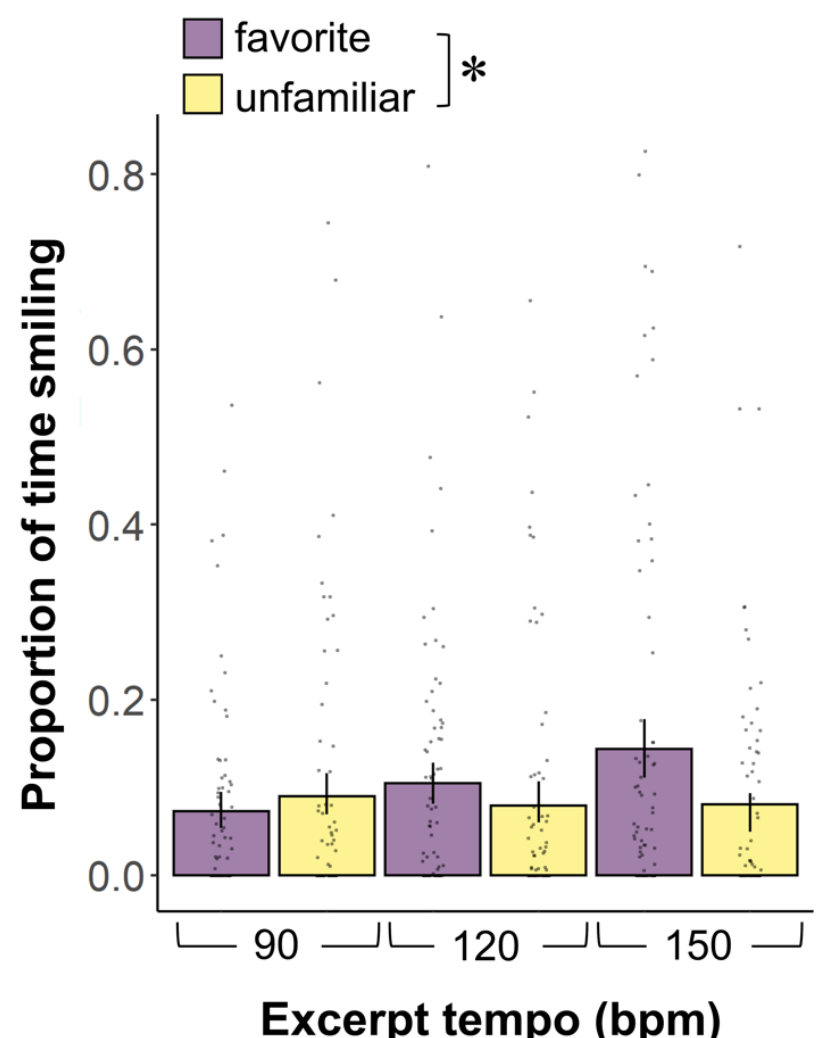

10 Figure 4. Depicts the proportion of time participants spent smiling.

11 Individual points represent individual participants, bars represent group

12 averages, and error bars represent 95\% within-subject confidence

13 intervals. ${ }^{*} p<.05$ 


\section{Discussion}

Prominent theories of rhythm perception argue that the motor system plays a significant role (e.g., Patel \& Iverson, 2014). Previous studies of caregiver-reported "dance" in infancy, as well as lab studies of infants' rhythmic capabilities, suggest that the motor system is stimulated by auditory input early in life. Here we observed that 1- to 3-year-old children demonstrate tempo flexibility when free dancing in a naturalistic home setting. Favorite music also elicited faster dancing than unfamiliar music across tempo conditions, and more dancing specifically in the slowest tempo condition (90 bpm). Children also smiled more frequently in response to their favorite compared to unfamiliar music, even though the favorite music was played at a different tempo from what they were used to hearing. Paralleling laboratory research with younger infants (Kragness, Johnson, \& Cirelli, 2022), we also found that children smiled more frequently on trials when they danced more, highlighting the connection between musical movement and expressions of joy in early life.

Many studies examining auditory-motor abilities in adults involve highly constrained tasks which require participants to clap, tap, or drum along to a beat or metronome (see Repp \& Su, 2013 for a review). Because these tasks are clearly inappropriate for young children and infants, investigations with these populations have opted to use bell-ringing, drumming, or motion capture of spontaneous movements (Rocha \& Mareschal, 2017; Yu \& Myowa, 2021; Zentner \& Eerola, 2010). The current work points to home-based recordings of free dancing as a fruitful methodology for investigating early musical movements. Children are recorded in the comfort of their homes, at a time that is convenient for their mood and compliance, using only equipment commonly found in typical Canadian households. Nearly $90 \%$ of participants danced at some point in the home-based experiment, compared to only $\sim 60 \%$ of children in a previous free dancing study conducted in the lab (Eerola et al., 2006). This home-recording method is easy for caregivers to implement, likely accessible to a more diverse sample of families, and may improve children's motivation to demonstrate their abilities. 
Children in this study demonstrated tempo flexibility in their free dancing - that is, they

2 danced faster to the faster music than to the slower music. An open question is whether this

3 was driven by beat perception. Beat perception involves extracting an underlying, regular pulse

4 from the musical rhythm, and is characterized by the ability to form predictions about when

5 musical events should occur. Neural tracking of the beat appears to emerge early in life:

6 sleeping newborns and alert 7-month-old infants' neural responses appear to reflect pre-

7 attentive beat tracking when listening to auditory rhythmic sequences (Cirelli et al., 2016; Háden

8 et al., 2015; Winkler et al., 2009). By 2 to 4 months of age, infants can detect a $15 \%$ shift in

9 tempo in a 100 bpm isochronous auditory sequences (Baruch \& Drake, 1997). The early tempo

10 flexibility demonstrated here may reflect beat perception, but could alternatively be driven by

11 stimulus features, such as event density. Future studies could manipulate both tempo and event

12 density using novel composed rhythms to examine the relative influence of each on children's

13 musical movements.

14 Previous work with bell ringing and finger tapping suggests that by 1.5 years of age, 15 children's spontaneous motor tempo is roughly $120 \mathrm{bpm}$, increasing to about $150 \mathrm{bpm}$ and by

163.0 years (Rocha et al., 2021) before slowing across childhood and adulthood (Drake et al.,

17 2000; McAuley et al., 2006). Adults show more synchronization accuracy when moving to music

18 that is closer to their spontaneous motor tempo (Delevoye-Turrell et al., 2014). If children's

19 spontaneous motor tempo influences movement accuracy, we would expect that children's

20 movement tempos here would be more similar to the target tempos in the faster music

21 conditions, but found no evidence to support this prediction. Across tempo conditions, the rate

22 of children's movements corresponded to the beat rate, half beat rate, or double beat rate about

$2330 \%$ of the time. We also found evidence that the older children in our sample danced slower on

24 average than the younger children. One important consideration is that our children always

25 danced with music, which might elicit a qualitatively different response from moving rhythmically 
1 in silence. Alternatively, children's full-body spontaneous motor tempo may be quite different

2 from their spontaneous motor tempos during bell-ringing or finger tapping (Rocha et al., 2021). Annotators observed a wide range of dance "moves" - for example, twirls, claps, wiggles,

4 twists, bounces, and arm-waves. These self-selected "moves" that children produce when free

5 dancing might also provide a unique window into auditory-motor development. Children may be

6 better able to demonstrate their auditory-motor synchronization abilities with actions they are

7 comfortable producing. Across childhood, both gross and fine motor skills are highly in flux. As

8 children mature, they may find it pleasantly challenging to incorporate new body parts into their

9 dance repertoire. Age-related increases in dance complexity may explain why we did not

10 observe increases in tempo flexibility across our tested age range, such as those seen when

11 children across the age range are encouraged to perform the same action (Provasi \& Bobin-

12 Bègue, 2003; Rocha \& Mareschal, 2017; Yu \& Myowa, 2021). In future research, we plan to use

13 this home-recording method to investigate patterns of self-selected dance movements and

14 increasing dance complexity across childhood.

15 In one previous case study of a dancing toddler, V (Cirelli \& Trehub, 2018), researchers

16 observed dancing closer to the target tempo for unfamiliar music, with favorite music eliciting

17 fast dancing regardless of tempo. They hypothesized that the stimulating effects of favorite

18 music may have overridden tempo flexibility. In the present study, we also observed that favorite

19 music elicited more smiling and faster dancing across tempo conditions, supporting this

20 proposal. In some contexts, this increased arousal may artificially bring children closer or farther

21 to the target tempo depending on their prior level of physiological arousal, musical tempo, and

22 the child's individualized spontaneous motor tempo. We found only suggestive evidence that

23 tempo matching differed as a function of musical familiarity. Exploratory analyses revealed that

24 children were further from the target tempo for favorite music played at $120 \mathrm{bpm}$. While we

25 caution against over-interpreting this exploratory result, this tempo condition was often closest

26 to the original tempo $(M$ original tempo $=115 \mathrm{bpm})$, and therefore arguably the most familiar 
1 version of the favorite music. This may have made it especially exciting, which may have

2 detracted from tempo matching much like what was observed with V (Cirelli \& Trehub, 2018).

3 Children may have also demonstrated more joy and faster dancing for their favorite

4 music because they likely have extensive prior experience listening to and moving to these

5 pieces of music in social contexts. By age 4, children show better beat synchronization when

6 drumming with an adult partner (Kirschner \& Tomasello, 2009). Here, caregivers were instructed

7 to refrain from dancing with their child. In future research, it would be interesting to explore

8 whether moving with a partner to favorite compared to unfamiliar music differentially influences

9 tempo alignment.

10 These findings contribute to a growing body of work documenting the social-emotional

11 importance of familiar music in early childhood, and the flexibility with which young children can

12 identify familiar music. For example, 9- to 12-month-old infants listen longer and move

13 rhythmically more when listening to familiar songs, whether they are sung by their own caregiver

14 or by a stranger (Kragness, Johnson, \& Cirelli, 2022), and toddlers help a stranger more if they

15 are familiar with a song she is singing (Cirelli \& Trehub, 2018). Songs are proposed to be strong

16 signals for shared culture and group belonging, and this early responsiveness to favorite songs

17 suggests that young children already integrate these songs into their musical identities.

Intervention studies harnessing rhythmic coordination are gaining attention as avenues

19 to facilitate language abilities, social abilities, and self-regulation skills in children with hearing

20 loss, developmental disorders, and socio-economic risk factors (Hidalgo et al., 2017; Morris et

21 al., 2021; Williams \& Berthelsen, 2019). The results presented here suggest that children may

22 be more engaged with interventions that include familiar or preferred music. Intervention

23 planning should also consider encouraging both whole body free dancing and discrete

24 movements, such as drumming. Free dance may be especially fruitful for the youngest toddlers.

25 Notably, even in laboratory drumming studies with toddlers, younger children are more likely to

26 make extraneous "free dance" movements (such as bouncing and nodding) than older children 
1 (Rocha \& Mareschal, 2017; Yu \& Myowa, 2021). These free dancing movements, even when

2 they are considered extraneous to the task at hand, may themselves contain useful information

3 about the nature of early auditory-motor integration.

4

References

6 Aschersleben, G. (2002). Temporal control of movements in sensorimotor synchronization.

7 Brain and Cognition, 48(1), 66-79. https://doi.org/10.1006/brcg.2001.1304

8 Audacity Team. (2018). Audacity. [Computer software.]

9 Baruch, C., \& Drake, C. (1997). Tempo discrimination in infants. Infant Behavior and

11 Bergelson, E., Amatuni, A., Dailey, S., Koorathota, S., \& Tor, S. (2019). Day by day, hour by hour: Naturalistic language input to infants. Developmental Science, 22(1), e12715.

14 Brooks, M.E., Kristensen, K., van Benthem, K.J., Magnusson, A., Berg, C.W., Nielsen, A., Skaug, H.J., Maechler, M., Bolker, B.M. (2017). glmmTMB balances speed and flexibility among packages for zero-inflated generalized linear mixed modeling. The $R$

17
Journal, 9(2), 378-400. https://journal.r-project.org/archive/2017/RJ-2017-

066/index.html.

Cirelli, L. K., Spinelli, C., Nozaradan, S., \& Trainor, L. J. (2016). Measuring neural entrainment to beat and meter in infants: Effects of music background. Frontiers in Neuroscience, 10, Article 229. https://doi.org/10.3389/fnins.2016.00229

2 Cirelli, L. K., \& Trehub, S. E. (2018). Infants help singers of familiar songs. Music \& Science, 1. https://doi.org/10.1177/2059204318761622

24 Cirelli, L. K., \& Trehub, S. E. (2019). Dancing to Metallica and Dora: Case study of a 19-month25 old. Frontiers in Psychology, 10, 1073. https://doi.org/10.3389/fpsyg.2019.01073 
1 Cirelli, L. K., Peiris, R., Tavassoli, N., Recchia, H., \& Ross, H. (2020). It takes two to tango: preschool siblings' musical play and prosociality in the home. Social Development, 29(4), 964-975. https://doi.org/10.1111/sode.12439

Cirelli, L. K., \& Trehub, S. E. (2020). Familiar songs reduce infant distress. Developmental Psychology, 56(5), 861-868. https://doi.org/10.1037/dev0000917

Cannon, J. J., \& Patel, A. D. (2021). How beat perception co-opts motor neurophysiology. Trends in Cognitive Sciences, 25(2), 137-150. https://doi.org/10.1016/j.tics.2020.11.002

Delevoye-Turrell, Y., Dione, M., \& Agneray, G. (2014). Spontaneous motor tempo is the easiest pace to act upon for both the emergent and the predictive timing modes. Procedia-Social and Behavioral Sciences, 126, 121-122. https://doi.org/10.1016/j.sbspro.2014.02.338

Drake, C., Jones, M. R., \& Baruch, C. (2000). The development of rhythmic attending in auditory sequences: attunement, referent period, focal attending. Cognition, 77(3), 251-288. https://doi.org/10.1016/S0010-0277(00)00106-2

de Kock, R., Gladhill, K. A., Ali, M. N., Joiner, W. M., \& Wiener, M. (2021). How movements shape the perception of time. Trends in Cognitive Sciences, S1364661321002059. https://doi.org/10.1016/i.tics.2021.08.002

Eerola, T., Luck, G., \& Toiviainen, P. (2006). An investigation of preschoolers' corporeal synchronization with music. In M. Baroni, A. R. Addessi, R. Caterina, \& M. Costa (Eds.), Proceedings of the 9th International Conference on Music Perception and Cognition (CD-ROM (pp. 472-476). Bologna, Italy: Università di Bologna

Fujii, S., Watanabe, H., Oohashi, H., Hirashima, M., Nozaki, D., \& Taga, G. (2014). Precursors of dancing and singing to music in three- to four-months-old infants. PLOS ONE, 9(5), e97680. https://doi.org/10.1371/journal.pone.0097680

Gordon, C. L., Cobb, P. R., \& Balasubramanian, R. (2018). Recruitment of the motor system during music listening: An ALE meta-analysis of fMRI data. PLOS ONE, 13(11). https://doi.org/10.1371/journal.pone.0207213 
1 Grahn, J. A., \& Brett, M. (2007). Rhythm and beat perception in motor areas of the brain. Journal of Cognitive Neuroscience, 19(5), 893-906. https://doi.org/10.1162/jocn.2007.19.5.893

Gudmundsdottir, H., \& Trehub, S. (2018). Adults recognize toddlers' song renditions. Psychology of Music, 46(2), 281-291. https://doi.org/10.1177/0305735617711762

Háden, G. P., Honing, H., Török, M., \& Winkler, I. (2015). Detecting the temporal structure of sound sequences in newborn infants. International Journal of Psychophysiology, 96(1), 23-28. https://doi.org/10.1016/j.ijpsycho.2015.02.024

Henry, M. J., Cook, P. F., de Reus, K., Nityananda, V., Rouse, A. A., \& Kotz, S. A. (2021). An ecological approach to measuring synchronization abilities across the animal kingdom. Philosophical Transactions of the Royal Society B: Biological Sciences, 376(1835), 20200336. https://doi.org/10.1098/rstb.2020.0336

Herzberg, O., Fletcher, K. K., Schatz, J. L., Adolph, K. E., \& Tamis-LeMonda, C. S. (2021). Infant exuberant object play at home: Immense amounts of time-distributed, variable practice. Child Development. Advance online publication. https://doi.org/10.1111/cdev.13669

Hidalgo, C., Falk, S., \& Schön, D. (2017). Speak on time! Effects of a musical rhythmic training on children with hearing loss. Hearing Research, 351, 11-18. https://doi.org/10.1016/i.heares.2017.05.006

Kim, M. \& Schachner, A. (2021, March 607). The origins of dance: Characterizing the development of dance during the first two years of life. [Conference presentation]. Future Directions of Music Cognition, online. https://osf.io/xerwd/

Kirschner, S., \& Tomasello, M. (2009). Joint drumming: Social context facilitates synchronization in preschool children. Journal of Experimental Child Psychology, 102(3), 299-314. https://doi.org/10.1016/j.jecp.2008.07.005 
1 Kragness, H. E., Anderson, L., Chow, E., Schmuckler, M., \& Cirelli, L. K. (2022). Musical groove shapes children's free dancing. PsyArXiv. https://doi.org/10.31234/osf.io/6dgpj

3 Kragness, H. E., Ullah, F., Chan, E., Moses, R., \& Cirelli, L. (2022). Tiny dancers: Effects of musical familiarity and tempo on children's free dancing [Data set and code book]. Open Science Framework. Retrieved from osf.io/q8zjy

6 Kragness, H. E., Ullah, F., Chan, E., Moses, R., \& Cirelli, L. (2020). Tiny dancers: Effects of musical familiarity and tempo on children's free dancing [Data set]. Databrary. Retrieved from http://doi.org/10.17910/b7.1141.

9 Kragness, H. E., Johnson, E. K., \& Cirelli, L. K. (2022). The song, not the singer: Infants prefer to listen to familiar songs, regardless of singer identity. Developmental Science, 25, Article e13149. http://doi.org/10.1111/desc.13149 system. Behavior Research Methods, 41, 841-849. https://doi.org/10.3758/BRM.41.3.841

Lenth, R. (2020). emmeans: Estimated Marginal Means, aka Least-Squares Means. R package version 1.4.5. https://CRAN.R-project.org/package=emmeans

17 Mathis, A., Mamidanna, P., Cury, K.M., Abe, T., Murthy, V. N., Mathis, M. W., \& Bethge, M. (2018). DeepLabCut: markerless pose estimation of user-defined body parts with deep learning. Nature Neuroscience, 21 (9). 1281-1289 (2018). https://doi.org/10.1038/s41593-018-0209-y

21 Manning, F., \& Schutz, M. (2013). "Moving to the beat" improves timing perception. Psychonomic Bulletin \& Review, 20(6), 1133-1139. https://doi.org/10.3758/s13423-013- 
1 McAuley, J. D., Jones, M. R., Holub, S., Johnston, H. M., \& Miller, N. S. (2006). The time of our lives: Life span development of timing and event tracking. Journal of Experimental Psychology: General, 135(3), 348-367. https://doi.org/10.1037/0096-3445.135.3.348

4 Merchant, H., \& Honing, H. (2014). Are non-human primates capable of rhythmic entrainment?

$5 \quad$ Evidence for the gradual audiomotor evolution hypothesis. Frontiers in Neuroscience, 7. $6 \quad$ https://doi.org/10.3389/fnins.2013.00274

7 Morillon, B., \& Baillet, S. (2017). Motor origin of temporal predictions in auditory attention.

$8 \quad$ Nature Communications, 5(1). https://doi.org/10.1038/ncomms6255

9 Morris, P., Hope, E., Foulsham, T., \& Mills, J. P. (2020, November 17). The effectiveness of mirroring- and rhythm-based interventions for children diagnosed with Autism Spectrum

Nave-Blodgett, J. E., Snyder, J. S., \& Hannon, E. E. (2020). Hierarchical beat perception develops throughout childhood and adolescence and is enhanced in those with musical training. Journal of Experimental Psychology: General, 150(2), 314-339. https://doi.org/10.1037/xge0000903

Patel, A. D., \& Iversen, J. R. (2014). The evolutionary neuroscience of musical beat perception: The Action Simulation for Auditory Prediction (ASAP) hypothesis. Frontiers in Systems Neuroscience, 8. https://doi.org/10.3389/fnsys.2014.00057

Phillips-Silver, J., \& Trainor, L. J. (2005). Feeling the beat: movement influences infant rhythm perception. Science, 308(5727), 1430. https://doi.org/10.1126/science.1110922

Politimou, N., Stewart, L., Müllensiefen, D., \& Franco, F. (2018). Music@ Home: A novel instrument to assess the home musical environment in the early years. PloS one, 13(4), Article e0193819. https://doi.org/10.1371/journal.pone.0193819

Provasi, J., \& Bobin-Bègue, A. (2003). Spontaneous motor tempo and rhythmical synchronisation in 21/2- and 4-year-old children. International Journal of Behavioral Development, 27(3), 220-231. https://doi.org/10.1080/01650250244000290 
1 R Core Team (2020). R: A language and environment for statistical computing. R Foundation for Statistical Computing, Vienna, Austria. URL https://www.R-project.org/.

3 Repp, B. H. (2005). Sensorimotor synchronization: A review of the tapping literature. Psychonomic Bulletin \& Review, 12(6), 969-992. https://doi.org/10.3758/bf03206433

5 Repp, B. H., \& Su, Y.-H. (2013). Sensorimotor synchronization: A review of recent research (2006-2012). Psychonomic Bulletin \& Review, 20(3), 403-452. https://doi.org/10.3758/s13423-012-0371-2

8 Rocha, S., \& Mareschal, D. (2017). Getting into the groove: The development of tempoflexibility between 10 and 18 months. Infancy, 22(4), 540-551. https://doi.org/10.1111/infa.12169

Rocha, S., Southgate, V., \& Mareschal, D. (2021). Infant spontaneous motor tempo. Developmental Science, 24(2), Article e13032. https://doi.org/10.1111/desc.13032

Savage, P. E., Brown, S., Sakai, E., \& Currie, T. E. (2015). Statistical universals reveal the structures and functions of human music. Proceedings of the National Academy of Sciences, 112(29), 8987-8992. https://doi.org/10.1073/pnas.1414495112

Scott, K. M., Chu, J., and Schulz, L. E. (2017). Lookit (part 2): Assessing the viability of online developmental research, results from three case studies. Open Mind 1(1), 15-29. https://doi.org/10.1162/opmi a 00001

Slone, L. K., Abney, D. H., Borjon, J. I., Chen, C. H., Franchak, J. M., Pearcy, D., SuarezRivera, C., Xu, T. L., Zhang, Y., Smith, L. B \& Yu, C. (2018). Gaze in action: Headmounted eye tracking of children's dynamic visual attention during naturalistic behavior. Journal of Visualized Experiments: JoVE, 141. https://doi.org/10.3791/58496

Trehub, S. E., \& Gudmundsdottir, H. R. (2019). Mothers as singing mentors for infants. In G. F. Welch, D. M. Howard, \& J. Nix (Eds.), The Oxford Handbook of Singing (pp. 454-470). Oxford University Press. https://doi.org/10.1093/oxfordhb/9780199660773.013.25 
1 Williams, K. E., \& Berthelsen, D. (2019). Implementation of a rhythm and movement intervention

2 to support self-regulation skills of preschool-aged children in disadvantaged

3 communities. Psychology of Music, 47(6), 800-820.

$4 \quad$ https://doi.org/10.1177/0305735619861433

5 Winkler, I., Háden, G. P., Ladinig, O., Sziller, I., \& Honing, H. (2009). Newborn infants detect the 6 beat in music. Proceedings of the National Academy of Sciences, 106(7), 2468-2471.

7 https://doi.org/10.1073/pnas.0809035106

8 Yu, L., \& Myowa, M. (2021). The early development of tempo adjustment and synchronization

9 during joint drumming: A study of 18- to 42-month-old children. Infancy, 26(4), 635-646.

$10 \quad$ https://doi.org/10.1111/infa. 12403

11 Zarco, W., Merchant, H., Prado, L., \& Mendez, J. C. (2009). Subsecond timing in primates:

12 Comparison of interval production between human subjects and rhesus monkeys.

13 Journal of Neurophysiology, 102(6), 3191-3202. https://doi.org/10.1152/jn.00066.2009

14 Zentner, M., \& Eerola, T. (2010). Rhythmic engagement with music in infancy. Proceedings of 15 the National Academy of Sciences, 107(13), 5768-5773.

$16 \quad$ https://doi.org/10.1073/pnas.1000121107 